ORIGINAL ARTICLE

\title{
Medication errors in intravenous drug preparation and administration: a multicentre audit in the UK, Germany and France
}

\author{
D H Cousins, B Sabatier, D Begue, C Schmitt, T Hoppe-Tichy
}

Qual Saf Health Care 2005;14:190-195. doi: 10.1136/qshc.2003.006676

Background: Previous studies have identified medication errors in preparing and administering intravenous medicines of $13-84 \%$ in hospitals in individual countries.

Objective: To compare the effect of the design and implementation of systems for the preparation and administration of intravenous therapy in hospitals in three European countries on the number of observed medication errors. To gain a better understanding of these risks and the methods used in each country to manage them.

Design: Prospective audit.

Setting: Six hospital departments in the UK, Germany and France willing to participate in the audit as part of a quality improvement programme.

Methods: Direct observation of the preparation and the administration of intravenous drugs made by a single observer in each country.

See end of article for authors' affiliations Main outcome measures: Medication process errors.

.....................

Correspondence to: Professor D H Cousins, Pharmacy Academic Practice Unit, University of Derby, Mickleover, Derby DE3 5GX, UK;

DHCousins@nt|world.com

Accepted for publication 26 February 2005

Results: 824 doses were prepared and 798 doses administered. The product was either not labelled or incorrectly labelled in $43 \%, 99 \%$, and $20 \%$ of doses administered in the UK, German and French hospitals, respectively. The wrong diluent was used in $1 \%, 49 \%$ and $18 \%$ of cases, respectively, and the wrong rate of administration was selected for $49 \%, 21 \%$ and $5 \%$ of doses observed, respectively. At least one deviation from aseptic technique was observed among 100\%, 58\%, and 19\% of cases in the three countries.

Conclusion: Uncontrolled risks in the intravenous systems studied were observed in all three countries. Intravenous therapy must be regarded as a high risk activity where the use of risk management procedures to minimise risk to patients is seen as a high priority by all those involved with these duties. There is a requirement to develop better national (possibly international) procedures for safe intravenous practice.

! ntravenous therapy is a complex process usually requiring the preparation of the medicine in the clinical areas before administration to the patient. There have been reports of deaths and harm following medication errors such as wrong drug, dose, diluent, and cross contamination errors with intravenous therapy. ${ }^{2}$ There is a growing awareness of the importance of the design and implementation of systems of care on the risk of medication errors..$^{3-7}$ Previous studies have identified errors in preparing and administering intravenous medicines of $13-84 \%$ in hospitals within one country. ${ }^{8-19}$ One study has compared errors in two countries. ${ }^{20}$ The design of procedures and implementation of the system for preparing and administering intravenous medicines was thought likely to be influenced by national factors such as legislation, healthcare system requirements, professional standards, university course curricula, and delivery of education and training for healthcare staff. The objective of this audit of intravenous practice in study hospitals in the UK, France and Germany was to compare the effect of the design and implementation of their systems for the preparation and administration of intravenous therapy on the number of observed medication errors, and to gain a better understanding of theses risks and the methods used in each country to manage them.

\section{METHODS}

\section{Study centres}

The audit was performed in 2001-2002. The study centres were general medical and surgical wards in four general

hospitals in the UK, located in the Midlands area of England, three wards within the Heidelberg University Hospital in Germany (two surgical intensive care wards and one general surgical ward), and one department within the Hôpital Européen Georges Pompidou in Paris, France (Department of Clinical Immunology). Because the study was part of a quality improvement programme, the selection of centres was based on willingness to participate without any attempt at selecting a representative sample. In all these centres, hospital pharmacists displayed a strong interest in tracking intravenous medication errors, all had easy access to the medical departments, and all expressed a willingness both to conduct an audit of current practices and to have the results published. Agreement for the audit was obtained from hospital ethics committees in all hospitals.

Review of hospital intravenous therapy procedures Before undertaking the observation the observer reviewed the hospital written intravenous therapy procedures. In UK hospitals, because of difficulties in developing and maintaining written procedures, hospitals had adopted the use of the procedures in a nationally published Manual of Clinical Nursing Procedures. ${ }^{21}$ In the hospitals in Germany and France there were also difficulties in developing and maintaining written procedures; some ward areas had developed their own, in other cases no formal written procedures were available, and there were no nationally published procedures. In all cases there was an assumption that the preparation and administration of intravenous medicines had been taught during 


\begin{tabular}{|c|c|c|c|c|}
\hline & UK wards & German wards & French ward & Total \\
\hline \multicolumn{5}{|l|}{ Observations } \\
\hline Preparations & 299 & 425 & 100 & 824 \\
\hline Administrations & 273 & 425 & 100 & 798 \\
\hline \multicolumn{5}{|l|}{ Ward type mix } \\
\hline General ward & 299 & 82 & 100 & 481 \\
\hline Intensive care & 0 & 343 & 0 & 343 \\
\hline \multicolumn{5}{|l|}{ Nurses } \\
\hline Total number & 122 & NC & 22 & 144 \\
\hline Registered (years) & 10.8 & NC & 8.9 & \\
\hline Accredited for IV therapy (years) & 6.4 & NC & NC & \\
\hline \multicolumn{5}{|l|}{ Type of intravenous products prepared } \\
\hline Liquid into syringe & 58 & 271 & 2 & 332 \\
\hline Liquid into bag & 13 & 73 & 25 & 111 \\
\hline Powder into syringe & 95 & 30 & 1 & 126 \\
\hline Powder into bag & 104 & 26 & 72 & 202 \\
\hline Pharmacy or industry prepared & 29 & 25 & 0 & 54 \\
\hline
\end{tabular}

basic training within medical and nursing schools and this information was supplemented by written procedures developed or endorsed by the hospital.

\section{Definition of intravenous medication error}

We defined an intravenous drug error as a deviation in the preparation or administration of a medicine from a doctor's prescription, hospital intravenous procedures, or the manufacturer's instructions. The clinical appropriateness of the prescription and the potential clinical outcomes resulting from the observed medication error were not recorded. Medication recorded in this study can therefore be regarded as process errors-for example, failure to label prepared products that were not used immediately and deviations of more than 1 hour from the prescribed administration time were considered as errors. Conversely, we excluded from our definition mistakes that were corrected by a member of staff before administration.

\section{Audit form}

An audit form based on procedures in the Manual of Clinical Nursing Procedures ${ }^{21}$ was piloted and tested in the UK and later adapted to Germany and France. It was designed to record all actions of the nursing staff from the time the intravenous drug prescription was received to the time it was delivered to patients. The following items were observed:

- qualifications of the staff member (no formal identification);

- whether the right drug was chosen;

- the right diluent (compatibility with drug and volume to achieve the right concentration);

- whether the preparation was mixed properly;

- the right dose delivered at the right time through the right route;

- the right infusion rate;

- details of aseptic method used (hands washed, preparation surface cleaned, vials and additive ports disinfected with alcohol impregnated swabs, sterile areas not touched); and

- correct labelling (identification of patient, drug, dose and time for administration).

\section{Observational audit method}

The observational audit method was preferred over the selfreporting of medication errors or the questionnaire survey method as it has been shown to provide the most reliable data. ${ }^{22-24}$ In each centre, data were collected by a single observer. The observer was present on the wards and accompanied and observed the preparation and administration of intravenous doses each day excluding weekends. In the UK and in France the observer was a pharmacy technician. In Germany the observer was a pharmacy student in practical training. Information about the objective of the study was shared with all the staff in the UK but the reason for the audit was disguised from the nursing staff being observed in the hospitals in France and Germany. The observer was instructed not to intervene when an error was detected except if the error could be the cause of a serious adverse event. In the UK the data collection took between 5 and 6 weeks, with the pharmacy technician being present on the ward between 07.30 hours and 19.00 hours. In Germany the observer was present every day between 10.00 hours and 15.00 hours for 6 weeks. In France the pharmacy technician was on the ward from 09.00 hours to 16.15 hours for 3 months. The results of the study were fed back to the participating nurses. In Germany the results were published in two German journals. ${ }^{25} 26$

\section{Data analysis}

Observational data were entered into computer software spreadsheet applications and analysed in each country before being pooled together to enable international comparisons.

\section{RESULTS}

\section{Characteristics of study centres}

The main characteristics of the observations performed are summarised in table 1. A total of 824 doses prepared and 798 doses administered were observed.

In the UK 299 doses were observed being prepared and 273 doses were observed being administered in general wards of the four hospitals. Not all prepared doses were observed being administered either because the intravenous route was no longer available or the patient was off the ward visiting other departments. One hundred and twenty two nurses were observed in the study. The average length of time since registration was 10.8 years and they had been accredited for IV therapy for 6.4 years.

In Germany a total of 425 observations were recorded, 343 $(81 \%)$ in the two surgical intensive care units and $82(19 \%)$ in the general surgical wards. No information about nurses could be collected because it was too sensitive.

In France 100 doses were observed being prepared and administered in the department of clinical immunology by 13 registered nurses with an average length of time since registration of 8.9 years seniority and nine nurse trainees. 
Table 2 Number (\%) of labelling errors

\begin{tabular}{llll}
\hline & $\begin{array}{l}\text { UK wards } \\
(\mathbf{N}=\mathbf{2 7 3})\end{array}$ & $\begin{array}{l}\text { German wards } \\
(\mathbf{N}=\mathbf{4 2 5})\end{array}$ & $\begin{array}{l}\text { French ward } \\
(\mathbf{N}=100)\end{array}$ \\
\hline Any labelling error & $118(43 \%)$ & $421(99 \%)$ & $20(20 \%)$ \\
Name of drug missing & NC & $82(19 \%)$ & $13(13 \%)$ \\
Dose missing & NC & $125(29 \%)$ & $8(8 \%)$ \\
Name of patient missing & NC & $421(99 \%)$ & $13(13 \%)$ \\
Time of preparation missing & NC & $412(97 \%)$ & $9(9 \%)$ \\
Label absent or incomplete and & $55(20 \%)$ & $171(40 \%)$ & NC \\
dose not administered immediately & & & \\
\hline NC, data not collected. & & & \\
\hline
\end{tabular}

Information about the type of intravenous formulations observed, grouped into five categories, are shown in table 1. A minority of the observations derived from ready to use products, whether pharmacy or industry prepared, with 29 (10\%) such observations in the UK hospitals and $25(6 \%)$ in the German wards. Industry prepared ready to use products were available in only two out of the four hospitals in the UK.

\section{Incomplete labelling, wrong drug, and dose errors}

Lack of appropriate labelling was a frequent error (table 2). Labelling errors accounted for 118 of 273 observations (43\%) in the UK centres, 421 (99\%) in the German centre, and 20 $(20 \%)$ in the French centre. In 55 cases $(20 \%)$ in the UK hospitals, doses were administered 10 minutes or later after they had been prepared and were not labelled at all. Similarly, in the German wards 171 (40\%) of the doses with incomplete labels were not administered immediately after they had been prepared.

\section{Wrong diluent errors and wrong rate errors}

The most common error was administering the intravenous doses at the wrong rate, and usually too quickly (table 3 ). Errors of administration rate were 132 out of 273 (49\%) in the UK centres, 34 out of 161 observations $(21 \%)$ in the German hospital, and 5 out of 100 (5\%) in the French centre. The second most frequent deviation from recommendations was preparing the intravenous doses with the wrong diluent. There were two (1\%) such cases in the UK wards, 208 (49\%) in the German centre, and 18 (18\%) in the French hospital.

Other errors included not mixing products adequately as recommended ( 190 out of $240(79 \%)$ observations in German wards and one (1\%) in the French centre); administering the product too late or too early (49 (18\%) cases in UK hospitals, seven $(2 \%)$ in the German hospital) and four $(4 \%)$ in the French centre); preparing or infusing the wrong volume ( 13 $(2 \%)$ cases overall in all centres); and using the wrong route of administration (eight ( $1 \%$ ) cases overall). In the UK and in Germany no adverse events related to individual medication errors were reported. Errors relating to dosage were the possible cause for four adverse events $(4 \%)$ in the French hospital, possibly prolonging hospital stay in three cases.

\section{Aseptic methods}

Aseptic methods for preparing intravenous medicines are described in table 4. General recommendations include cleaning the preparation area, washing hands or wearing sterile gloves, disinfecting vials, ampoules and additive ports with alcohol impregnated swabs, and making sure that windows are closed in the preparation area. These recommendations were part of the hospital intravenous therapy policy in all centres. The preparation area was never cleaned in the UK hospitals $(0 \%)$, it was properly cleaned in $18(4 \%)$ cases in the German wards and in 81 cases $(81 \%)$ in the French centre. Hands were never washed $(0 \%)$ in any of the four hospitals in the UK nor were sterile gloves worn, but non-sterile gloves were worn in one hospital in order to protect nurses from the medicines. Hands were washed in the German hospital in 22 cases (5\%) and 91 preparations (91\%) observed in the French hospital. In the UK centres the tops of vials were wiped with alcohol impregnated swabs in four cases (1\%). In the German wards not all nurses considered it important to disinfect the vials, so that it was performed in only $180(42 \%)$ of cases. Vial tops were swabbed in the French hospital in 96 (96\%) of cases. In the preparation area either windows were closed or there were no windows at all in the majority of cases (557 of 724 observations (68\%) overall in the UK and German centres).

\section{DISCUSSION}

This study shows that current systems for intravenous therapy in the European hospitals studied do not minimise patient safety risks. Initiatives in individual hospitals or countries for one or two aspects of the process have reduced the risk, but overall all systems need to be improved.

\section{Limitations of study}

The limitations of our study are as follows:

- Self-selection of hospitals in individual countries to take part in the audit.

- Convenience sampling of clinical areas within each hospital.

Table 3 Number (\%) of observed medication errors

\begin{tabular}{llll}
\hline & $\begin{array}{l}\text { UK wards } \\
(\mathbf{N}=\mathbf{2 7 3})\end{array}$ & $\begin{array}{l}\text { German wards } \\
(\mathbf{N = 4 2 5 )}\end{array}$ & $\begin{array}{l}\text { French ward } \\
(\mathbf{N}=100)\end{array}$ \\
\hline Wrong drug & 0 & 0 & 0 \\
Wrong diluent & $2(1 \%)$ & $208(49 \%)$ & $18(18 \%)$ \\
Product not mixed & $\mathrm{NC}$ & $190 / 240(79 \%)$ & $1(1 \%)$ \\
Wrong dose or wrong & $1(1 \%)$ & $7(2 \%)$ & $5(5 \%)$ \\
infusion volume & $1(1 \%)$ & $6(1 \%)$ & $1(1 \%)$ \\
Wrong route & $132(48 \%)$ & $34 / 161(21 \%)$ & $5(5 \%)$ \\
Wrong administration rate & $79(18 \%)$ & $7(2 \%)$ & $4(4 \%)$ \\
Wrong time & & & \\
\hline NC, data not collected. & &
\end{tabular}


Table 4 Aseptic methods of preparation: values are numbers (percentages)

\begin{tabular}{llll}
\hline & $\begin{array}{l}\text { UK wards } \\
(\mathbf{N}=\mathbf{2 9 9})\end{array}$ & $\begin{array}{l}\text { German wards } \\
\mathbf{N}=\mathbf{4 2 5})\end{array}$ & $\begin{array}{l}\text { French ward } \\
(\mathbf{N}=100)\end{array}$ \\
\hline Cleaned preparation area & $0(0)$ & $18(4 \%)$ & $81(81 \%)$ \\
Hands washed & $0(0)$ & $22(5 \%)$ & $91(91 \%)$ \\
Swab vial top & $4(1 \%)$ & $180(42 \%)$ & $96(96 \%)$ \\
Window closed in preparation area & $213(71 \%)$ & $344(81 \%)$ & $\mathrm{NC}$ \\
\hline NC, data not collected. & & & \\
\hline
\end{tabular}

- Use of different observers in different countries. It was not possible to use the same observer(s) for all three countries and, although we tried to control this variable by agreeing the definition for medication error and using a standardised audit form, we did not attempt to assess observer reliability between the three countries.

- The failure to capture some data sets in some countries.

- Focus on process errors. Our method did not attempt to link the observed process errors to potential clinical outcomes. Some previous studies ${ }^{13} 27$ have established that some process errors may be associated with potentially severe clinical outcomes.

\section{Intravenous procedures}

In all three countries difficulties were encountered by hospitals in developing and maintaining intravenous procedures. Without agreed procedures of this type it is very difficult to teach, maintain, and audit safe practice. The intravenous procedures in the Manual of Clinical Nursing Procedures $^{21}$ in the UK has recently been supplemented by a publication by the Royal College of Nursing (Standards for Infusion Therapy published in 2003). ${ }^{28}$ The use of national (and professionally endorsed) procedures of this type is seen as very helpful in defining safe practice. However, individual institutions have a responsibility to audit the implementation of these procedures regularly and to provide feedback to practitioners on those areas of practice that are out of compliance with the procedure.

\section{Wrong drug and dose errors}

The results from the UK and German hospitals indicate that a significant percentage of products that are not labelled are not used immediately and are stored temporarily in the clinical area before administration. The temporary storage of unlabelled intravenous bolus medicines before administration has not been identified in previous research. We believe that this is an important risk that may cause the wrong drug or dose of a medicine to be administered. Practitioners intending to administer these products may be confused over the identity of the medicine, dose, or the intended patient to receive this medicine.

Reviewing written intravenous therapy procedures used in the study hospital indicates that these procedures provide no safe practice guidance on this issue. For example, in the Manual of Clinical Nursing Procedures in the UK, ${ }^{21}$ guidance is provided on labelling infusion fluids that are to be administered over several hours, but no recognition is given to the practice of preparing and temporarily storing intravenous medicines before bolus administration. There is an opportunity for hospital intravenous therapy procedures and training programmes to be strengthened by including the requirement to label medicines that are left unattended, even for short periods. In anaesthetic practice where intravenous bolus doses are administered from a range of syringes as an integral part of the anaesthetic process, a standard for syringe labelling in critical care areas has recently been introduced in the UK. ${ }^{29}$
There are also opportunities for the pharmaceutical industry to provide design solutions to assist practitioners to maintain and track product identity in clinical use. These designs include the use of ready to use pre-filled syringes and infusions, diluent products that can be permanently connected to drug vials during administration, ampoules and vials with flag labels; these labels can be transferred to easily label syringes and infusion bags.

\section{Wrong diluent errors}

In the German and French hospitals the most frequent error was preparing the medicine with the wrong diluent. The use of the wrong diluent may cause a reduction in the solubility of the medicine powder being reconstituted that can lead to powder particulates being administered to the patient. The use of the wrong diluent can also lead to a reduction in the stability and activity of the medicine and possible drug precipitation. The prescription for intravenous medicines seldom provides information concerning the diluent to be used to reconstitute or dilute the active intravenous medicine. The hospital intravenous therapy procedures indicate that healthcare staff preparing individual intravenous medicines in clinical areas should consult the manufacturer's product literature to obtain this information. Time constraints, custom and practice, and difficulties finding the relevant information within product literature are reasons frequently cited by practitioners why product information is seldom reviewed. Providing clinical staff with more readily available information concerning diluents may help to reduce the use of the wrong diluent. The clinical importance and use of this information can be reinforced as part of intravenous therapy training. This information could be added by the manufacturer or hospital pharmacy department as an additional warning label in large text to the outside of the medicine packaging. Quick reference tables could also be produced for individual hospitals or wards and displayed on the outside of ward medicine cupboards for easy access. Diluent products can also be supplied with medicines to provide a design solution to the problem. Pharmaceutical industry and/or hospital pharmacy departments could package these products together to assist practitioners to use the correct diluent with the medicine. Another alternative strategy is, where possible, to provide the intravenous product ready diluted and ready to administer to avoid diluent selection errors.

\section{Wrong rate errors}

In the UK hospitals the most frequent intravenous medication errors were related to the administration rate, usually higher than is recommended by manufacturers-for example, a $10 \mathrm{ml}$ dose of antibiotics administered by hand in a syringe in less than 1 minute instead of the 3-5 minutes recommended in the Specification of Product Characteristics (SPC), a result that is consistent with previous findings. ${ }^{10} 1226$ Whatever the administered drug characteristics, fast rates of drug administration are associated with pain, phlebitis, and loss of cannula patency. ${ }^{30}$ It could be observed that such errors were less frequent in the two hospitals 
equipped with CIVA and in the three hospitals that could provide industry prepared ready to use products. These types of products are presented in larger volumes to be infused by slow intravenous infusion. Once again, information concerning the rate of administration of intravenous bolus injections is seldom included in prescription details, and this information is not included in hospital intravenous therapy procedures. Practitioners are again expected to locate this information among the small print details in manufacturers' product information. Providing clinical staff with more readily available information concerning the safe rate of administration on medicine packaging or the use of quick reference tables may help to improve practice. Taxis and Barber in 2003 observed that, in some instances, the safe speed of injection of bolus doses was being deliberately violated because of lack of perceived risk, poor role models, and available technology. ${ }^{19}$

However, it may be argued that practitioners have insufficient time to administer intravenous bolus injections safely even when they are reminded of the correct administration rate. This observation is supported in the UK by the high percentage of wrong time errors (defined as administration of intravenous medicines 1 hour or more from the prescribed time). As this may indicate a high work load, wrong time errors are likely to be increased if nursing staff have to administer each bolus dose over 3-5 minutes as recommended in the product literature. If this is the case, then alternative methods of intravenous administration should be used such as short intravenous infusion therapy that does not require the practitioner to be at the bedside during the administration of the medicine.

\section{Aseptic methods}

Review of the written intravenous therapy procedures in the study hospitals revealed that aseptic requirements included hand washing before the procedure and cleaning ampoules, vials and intravenous infusion closures. These process errors associated with poor aseptic technique were the result of failure of the implementation of procedures rather than the availability or design of the procedures themselves. All nurses in the study had received training on intravenous therapy, although not all had received this training recently. Reason defines "violations" as deliberate-but not necessarily reprehensible-deviations from those practices deemed necessary by designers, managers, and regulatory agencies to maintain the safe operation of potentially hazardous systems. Reason subdivides this classification into routine and exceptional violation categories. The frequency with which aseptic process errors were observed in the study hospitals in the UK and Germany indicate that these were routine violations.

The importance of the safety procedure needs to be apparent to the operators, and there is little research demonstrating the importance of infection controls during the preparation of intravenous medicines in ward areas. There is a general assumption made by operators that the intravenous medicines are being prepared for immediate use and that the risk of infection is small. However, recent research in a German hospital following the deaths of two patients from meningitis caused by contamination of contrast media found other contaminated multiple dose vials in ward areas, and poor handling and storage of these types of medicine. ${ }^{27}$

In the French centre the clinical staff had recently been updated on aseptic methods and following the diagnosis of Legionnaire's disease in nine patients of whom four eventually died. In this hospital the relevance and surveillance of aseptic safety controls had been re-established. Nurses in the French hospital were observed to clean the

\section{Key messages}

- Prepared intravenous medicines were left for short periods of time unlabelled before being administered to patients. This is a previously unidentified risk that may cause the wrong drug or dose of a medicine to be administered in error to a patient.

- A frequent error involved using the wrong diluent to prepare an intravenous medicine. This may cause difficulties in dissolving drug powders, inactivation, and possible precipitation of the drug out of solution.

- Intravenous bolus medicines required to be administered by hand in a syringe were frequently administered too quickly and this practice is associated with phlebitis and loss of cannula patency.

- Aseptic procedures required for the safe preparation of intravenous doses were frequently violated by staff who were not always aware of the clinical consequences of not following these procedures.

preparation area $(81 \%)$, wash hands $(91 \%)$, and swab vial tops $(96 \%)$ while results were much poorer in the UK and in the German centres with preparation area cleaned in $2 \%$ of cases overall, hands washed in $3 \%$ of cases, and vial tops swabbed in $1 \%$ of cases in the UK hospitals and $42 \%$ of cases in the German hospital.

In the UK the National Patient Safety Agency published a Patient Safety Alert in September 2004 entitled "Clean Your Hands" that recognised hands as a repository of microbes which cause infection and the practical difficulties that staff face cleaning their hands between patients. ${ }^{31}$ The Alert requires the National Health Service in England and Wales to install alcohol based hand rubs at points of care by April 2005. These hand rub products are quick to use and can be applied without interrupting work. This high profile campaign will alert UK healthcare staff to the importance of hand cleaning and should improve the frequency of hand cleaning before the preparation of intravenous medicines, but this will need to be regularly audited.

A training strategy is also required to reinforce the clinical need to implement the agreed aseptic controls when preparing intravenous medicines. In the UK hospital pharmacy technician staff preparing intravenous medicines in aseptic clean rooms require regular competency assessment of aseptic technique using a simulation technique with nutrient broth. ${ }^{32}$ Manipulations take place in the usual work environment and the products produced are incubated to determine the contamination rate. Staff who contaminate products in simulation are withdrawn from duty and are retrained and undertake additional testing until they are able to produce uncontaminated products. Although it is unlikely to be practical to assess the intravenous therapy aseptic technique of all nursing staff in a hospital, it would be possible for small numbers to be assessed randomlypossibly during training sessions-and summary information concerning the contamination rate to be fed back to all nursing staff to stress the clinical importance of good aseptic technique.

In conclusion, it has been worthwhile comparing the systems for intravenous medicine in hospitals in three European countries. We found uncontrolled risks in the intravenous systems studied in all three countries. Intravenous therapy must be regarded as a high risk activity where the use of risk management procedures to minimise risk to patients is seen as a high priority by all those involved 
with these duties. There is a requirement to develop better national (possibly international) procedures for safe intravenous practice. These procedures can be used to train, maintain, and audit practice.

\section{Authors' affiliations}

D H Cousins, University of Derby, Derby, UK

B Sabatier, D Begue, Hôpital Européen Georges Pompidou, Paris,

France

C Schmitt, MDS Pharma Services, Sèvres, France

T Hoppe-Tichy, Heidelberg University Hospital, Heidelberg, Germany

Supported by a grant from Baxter Healthcare International.

\section{REFERENCES}

1 Cousins DH, Upton DR. Medication error report 125: A patient dies following cross infection with falciparum malaria. Pharmacy in Practice 1999;9:220-2.

2 Cousins DH, Upton DR. Medication error 79: How to prevent IV medicine errors. Pharmacy in Practice 1997;7:310-1.

3 Reason J. Human error. Cambridge: Cambridge University Press, 1990.

4 Department of Health. An organisation with a memory. London: Department of Health, 2000

5 Department of Health. Building a safer NHS for patients. London: Department of Health, 2001

6 Department of Health. Building a safer NHS for patients. Improving safe medication practice. London: Department of Health, 2004

7 Department of Health. Design for safety. A system-wide design-led approach to tackling patient safety in the NHS. London: Department of Health, 2003.

8 Thur MP, Miller WA, Latiolais CJ. Medication errors in a nurse controlled parenteral admixture programme. Am J Hosp Pharm 1972;29:293-304.

9 Clark CM, Bailie GR, Whitaker AM, et al. Parenteral drug delivery: value for money? Pharm J 1986;236:453-5.

10 Cousins DH, Lee $M$, Stanaway $M$, et al. Implementation and evaluation of a centralised IV additive service for antibiotic injections. Pharm J 1989:HS14-6.

11 O'Hare MCB, Gallagher T, Shields MD. Errors in the administration of intravenous drugs. BMJ 1995;10:1536-7.

12 Hartley GM, Dhillon S. An observational study of the prescribing and administration of intravenous drugs in a general hospital. In J Pharm Pract 1998;6:38-45.

13 Tissot $\mathbf{E}$, Cornette $\mathrm{C}$, Demoly $\mathrm{P}$, et al. Medication errors at the administration stage in an intensive care unit. Intensive Care Med 1999;25:353-9.
14 Bruce J, Wong I. Parenteral drug administration errors by nursing staff on an acute medical admissions ward during day duty. Drug Saf 2001;24:855-62.

15 Belkacem K, Lepaux DJ, Oliger R. Medication error rate in the hospital setting: a pilot study at the Jury-les-Metz Hospital Center. Presse Med 2001:30:785-9.

16 Calabrese AD, Erstad BL, Brandl K, et al. Medication administration errors in adult patients in the ICU. Intensive Care Med 2001;27:1592-8.

17 Van den Bemt PM, Fijn R, van der Voort PH, et al. Frequency and determinants of drug administration errors in the intensive care unit. Crit Care Med 2002;30:846-50.

18 Taxis K, Barber N. Ethnographic study of incidence and severity of intravenous drug errors. BMJ 2003;326:684-8.

19 Taxis K, Barber N. Causes of intravenous medication errors an ethnographic study. Qual Saf Health Care 2003;12:343-7.

20 Wirtz V, Taxis K, Barber ND. An observational study of intravenous medication errors in the United Kingdom and in Germany. Pharm World Sci 2003;25:104-11.

21 Mallett J, Dougherty L. Manual of clinical nursing procedures, 5th edn. Oxford: Blackwell Publishing, 2000

22 Allan EL, Barker KN. Fundamentals of medication errors research. Am J Hosp Pharm 1990;47:555-71.

23 Flynn EA, Barker KN, Pepper GA, et al. Comparison of methods for detecting medication errors in 36 hospitals and skilled-nursing facilities. Am J Health Syst Pharm 2002;59:436-46.

24 Dean B, Barber N. Validity and reliability of observational methods for studying medication administration errors. Am J Health Syst Pharm 2001;58:54-9.

25 Hoppe-Tichy T, Noe-Schwenn S, Wahlig A, et al. Medikationsfehler bei der Applikation parenteraler Arzneimittel. Krankenhauspharmazie 2002;23:11-7.

26 Hoppe-Tichy T, Noe-Schwenn S, Wahlig A, et al. Medikationsfehler mit schlimmen Folgen. Pflege Aktuell 2002;56:469-71

27 Mattner F, Gastmeir P. Bacterial contamination of multiple-dose vials: a prevalence study. Am J Infect Control 2004;32:12-6.

28 Royal College of Nursing. Standards for infusion therapy. London, Royal College of Nursing, 2003 (available at www.rcn.org.uk).

29 Birks RJS. Syringe labelling an international standard. Anaesthesia 2003:58:515-9.

30 Backhouse CM, Ball BR, Booth S, et al. Particulate contaminants of intravenous medication and infusions. J Pharm Pharmacol 1987;39:241-5.

31 National Patient Safety Agency. Patient Safety Alert 04. Clean your hands campaign can save lives. London, National Patient Safety Agency, 2004 (available at www.npsa.nhs.uk).

32 Beaney AM. Quality assurance of aseptic preparation services, 3rd edn. London: The Pharmaceutical Press, 2001.

\section{bmjupdates+}

bmiupdates+ is a unique and free alerting service, designed to keep you up to date with the medical literature that is truly important to your practice.

bmjupdates + will alert you to important new research and will provide you with the best new evidence concerning important advances in health care, tailored to your medical interests and time demands.

Where does the information come from?

bmjupdates+ applies an expert critical appraisal filter to over 100 top medical journals A panel of over 2000 physicians find the few 'must read' studies for each area of clinical interest

Sign up to receive your tailored email alerts, searching access and more...

www.bmjupdates.com 